\title{
Research for the Leakage Prevention Design of Automobile Air
}

\section{Conditioning Refrigerant}

\author{
Jiangjing $^{1, a}$, Huangjian ${ }^{2, b}$ \\ ${ }^{1}$ Guangxi vocational and technical college, Nanning, Guangxi, 530226 \\ ${ }^{2}$ Guangxi electromechanical vocational and technical college, Nanning, Guangxi, 530226
}

Key Word: Automobile Air conditioning, Refrigerant Leakage, Design, Prevention Control

\begin{abstract}
The leakage of automobile air conditioning refrigerant would not only make the air conditioner malfunction but also cause the air pollution to the environment. Therefore, it's very important to prevent and control the refrigerant leakage. This article provides a design solution to both manual and auto air conditioner by analyzing the leakage failure modes. The research value and operability have be verified by actual test. It provides much reference value for automobile maker and manufactory on air conditioning refrigerant leakage design improvement.
\end{abstract}

\section{Automobile Air Conditioning Refrigerant Leakage Failure Modes and Harm}

Nowadays, automobile air conditioning system is a closed system which contains air conditioning compressor, condenser, storage tank, expansion valve (or expansion tube) and evaporator. The mechanism of this type of compression recycle conditioning system is to recycle the refrigerant in the conditioning tubes and realize the cooling effect by changing the form of refrigerant from liquid state to gaseous state. The leakage often occurred at the connection of compressor, expansion valve, evaporator, storage tank and tubes ${ }^{[1]}$.

Since the conditioning system is a closed recycle system, the movement of refrigerant and freezed oil is the key for cooling effect. So the requirement for the sealing of the recycle system is very restrict. Any parts in this system has leakage would cause conditioning system malfunction eventually. If the leakage is very tiny, the conditioning system would gradually decrease the cooling effect and finally lose the cooling function.

Whichever types of leakage failure modes would cause air conditioner malfunction if no prevention taken and then cause energy waste and negatively affect the conformable of automobile. Besides, the refrigerant leakage into atmosphere would generate greenhouse effect. And more seriously some automobile use R12 ingredient which would destroy the ozone layer and cause serious air pollution.

\section{Current Automobile Air Conditioning Refrigerant Control Strategy}

The common failure modes of automobile air conditioner are lack of cooling, continuous cooling, poor cooling, heavily noise and etc. And most of them are caused by the refrigerant leakage. Therefore, the common repair to air conditioner is to check leakage and fix leakage. In order to find the related leakage parts, various check methods and equipment had been designed and developed, such as electrical leakage detector, fluorescence leakage detector, pressure leakage detector and foam leakage detector and etc ${ }^{[2]}$. Generally speaking, if the conditioning system has tiny leakage, it 
would not draw the attention of driver. But when it affect the cooling effect, the driver would seek for a check. When a leakage part found, the repair provider would drain out all refrigerant and replace the failed part. After a re-check without any leakage, fill in the refrigerant. Some driver would not repair the air conditioner until all refrigerant leak out. And some repair provider can't find out the leakage part and just add refrigerant again and again which is a cheap fix way. All those repair strategy are passive way to control the refrigerant leakage which cause energy waste and air pollution. Therefore, a more energy saving and environmental protection control strategy is expected and welcomed.

\section{Leakage Prevention Design}

If the refrigerant leakage could be detected and trigger a warning signal, it would realize stopping refrigerant leakage and regain the refrigerant by the refrigerant recovery machine which definitely control the leakage of refrigerant and save the energy and reduce the air pollution.

Design Principle. Related document and research shows the intake pressure and temperature and the exhaust pressure and temperature of compressor of automobile air conditioner are the main parameter reflecting the amount of refrigerant. Using test to find out the relationship between the pressure and the amount of refrigerant. The amount of refrigerant is not only related to the pressure but also related to the superheat, as shown below ${ }^{[3]}$.
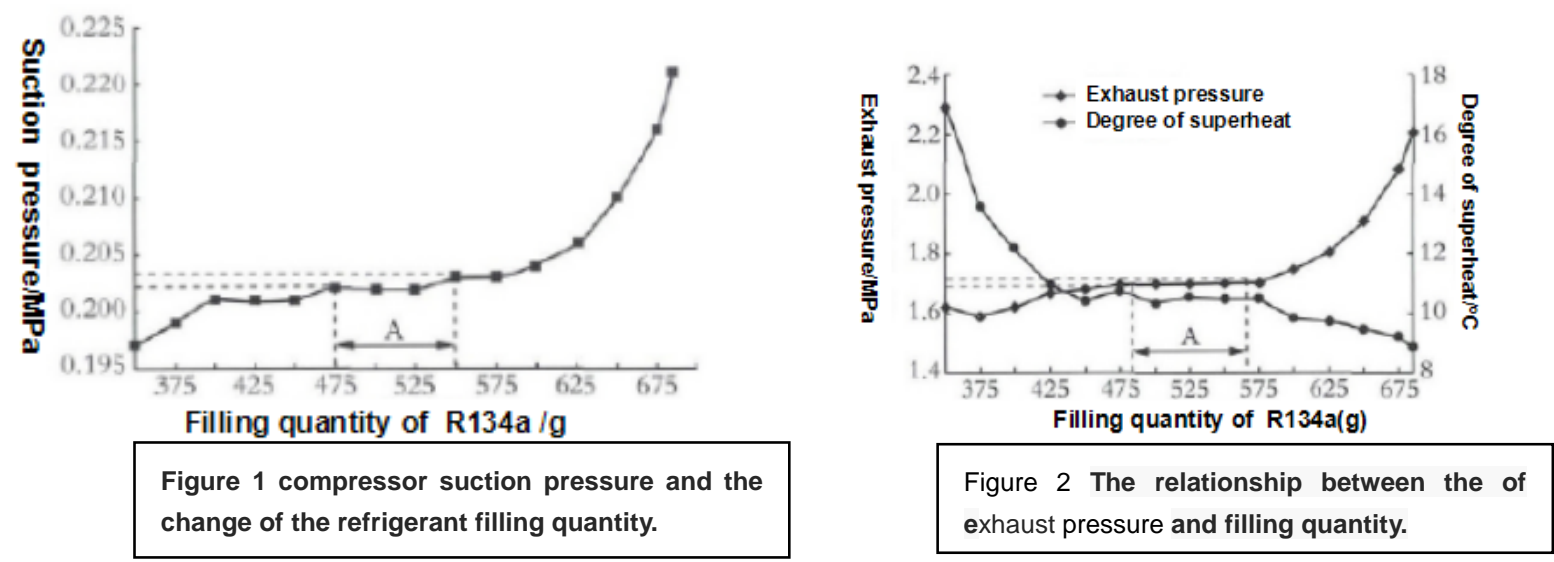

Based on the discrete points in the graph 1 and graph 2, using linear interpolation method,could get the amount of refrigerant in the intake pressure range of $0.197059 \mathrm{MPa} \sim 0.221176 \mathrm{MPa}$. Similarly, in the refrigeration system operation conditions, the amount of refrigerant corresponds to a static pressure when the air conditioner system reached to a balance. By measuring a range of amount and pressure data and linear interpolation method to get any R134a refrigerant amount to a pressure.

Design Method. To design a manual air condition control circuit without any pressure and temperature sensors, should replace the pressure sensing element like pressure switch with a pressure sensor and add related temperature sensor to get the ambient temperature. Also install a control ECU and actuators. To design a automatic air condition control circuit with pressure and temperature sensors, it could improve current design by adding control ECU and actuators. The design method is shown as following. 

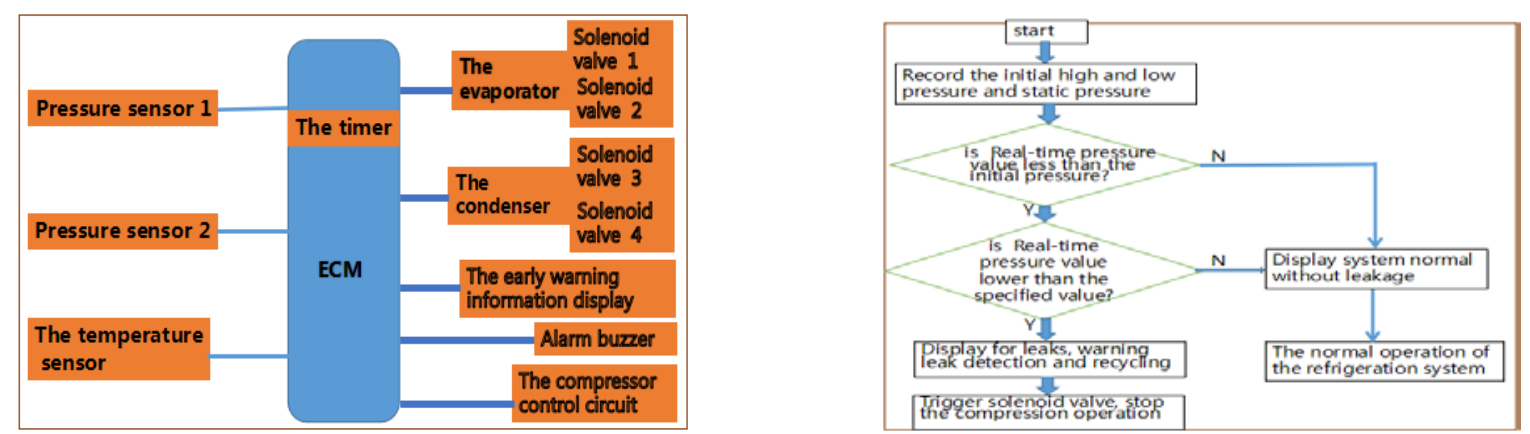

Figure 3 refrigerant leakage protection design Figure 4 refrigerant leakage protection control process

After the first time or vacuum of the conditioning system adding some refrigerant, the system recounts. Record the high pressure/low pressure and static pressure. When the real-time pressure below a predetermined value, even the air conditioner still work but the warning information is displayed and the buzzer is triggered. Once the pressure decreasing, send out signal to shut down four set electromagnetic valves and close the compressor circus, prevent the compressor operation to prevent the leakage. After the repair provider found out the leakage part and fixed, refill the refrigerant and continue to monitor the system and recount.

\section{Software Design}

Using the signal processing circuit to analyze the input information and using a microcontroller or an integrated circuit module to control the output signal. For the pressure monitoring of the conditioning system, it could be measured by using similar method of design of Tire Pressure Monitoring Technology ${ }^{[4]}$.The leakage prevention software design is as followings. Vacuum the conditioning system and refill the refrigerant to standard amount, operates normally, press the start button to work. At normal conditioning system operation, based on the pressure signal input to record the high/low pressure value and the static pressure when balance status. At the same time, the timer is on and compare the parameter once after an amount of time (several hours or one day) to judge if the real-time pressure below the initial pressure. If no pressure difference, the system shows no leakage. And then compare it with regular value, if not exceed the regular value then shows no leakage. If the real-time pressure is low and the pressure difference with the initial value is lower than the regular value, then shows leakage and reminder to check and recycling. And also trigger the electromagnetic valve to close the circus avoiding further leakage and stop the compressor. After the leakage fixed and refilled, the control system reset and recount.

\section{Conclusion}

During the automobile air conditioner operation, the pressure of the refrigerant varied according to the engine speed. When the conditioning compressor stop operation, the pressures of the refrigerant on the two sides of conditioning system would reach to a static equilibrium state. At this state, the pressure of high pressure side and low pressure side are equal. And the static pressure would varied by the amount of refrigerant. By using the difference method to find out the relationship between the static pressure and amount of refrigerant. Based on the detected signal of temperature, static pressure, the designed control system could notice the decrease of the static pressure by comparing the real-time signal and find out the leakage and send out warning information which would open the electromagnetic valve to prevent refrigerant leakage. And it also could reminder the operator to the recycle the refrigerant and repair the abnormal air conditioner which prevent progressive 
damage on the are conditioner and avoid refrigerant leakage, pollute the atmospheric environment and save the energy.

\section{References}

[1] Wang li. Automotive air conditioning leakage fault analysis and repair [J]. Journal of academy. Vocational education. 17, 2014.

[2] Vanessa. Introduction to automobile air conditioning system common fault elimination method. Heilongjiang science and technology information. Technology BBS. 2016.4.

[3] Jiang yichun. Refrigeration dose of automotive air conditioning system based on the real vehicle test bench and test study [J]. Journal of refrigeration and air conditioning. 2016.1. 16 (1).

[4] Qin Xiuchang. Tire pressure monitoring system (TPMS) in the research and design [D]. Sichuan: university of electronic science and technology. 2014.3 